\title{
Molecular identification of Fusarium spp. isolated from wheat based on sequencing of internal transcribed spacer (ITS) region
}

\section{Deyana Gencheva ${ }^{1 *}$, Georgi Beev ${ }^{2}$}

${ }^{1}$ Department of Genetics, Breeding and Reproduction, Faculty of Agriculture, Trakia University, Stara Zagora 6000, Bulgaria

2Department of Biochemistry, Microbiology and Physics, Faculty of Agricultural, Trakia University, Stara Zagora 6000, Bulgaria, e_mail: gbeev@abv.bg

*Corresponding author: Deyana Gencheva

Phone: +359 699 362, Email: dhristova@uni-sz.bg, ORCID ID: 0000-0003-2227-2245

\begin{abstract}
Molecular identification via Internal Transcribed Spacer region (nrDNA-ITS) sequencing of Fusarium spp. isolates from wheat originated from Stara Zagora region, were performed for the first time in Bulgaria. A total of 60 wheat samples (Triticum aestivum) were morphologically identified at the genus level as Fusarium spp. in advance. The rDNA-ITS region of all isolates was successfully amplified and the PCR products obtained were directly sequenced. After a comparison of detected sequences with NCBI database, members of three different fungal genera (Fusarium, Chaetomium, and Alternaria) were identified. Among Fusarium isolates, the F. tricinctum was prevailing, followed by F. poae. A total of three isolate F. proliferatum, F. graminearum and F. equiseti were presented with a single probe. The lowest genetic distance (0.004) was detected between F. tricinctum isolates. On the base of genetic distances, fungal isolates were grouped in two main clusters - one comprising F. tricinctum isolates and F. proliferatum, and second including $F$. equiseti, F. graminearum and F. poae. It could be concluded that the rDNA-ITS genome region of the genus Fusarium may be used as a suitable marker of early detection, accurate and reliable identification of Fusarium spp. contamination of wheat. The timely and accurate information would assist in the selection of appropriate approaches for control of fusarium infections and possible mycotoxins contamination of agricultural production.
\end{abstract}

Keywords: Fusarium spp., Triticum aestivum, Internal Transcribed Spacer region (rDNA-ITS), DNA sequencing

\section{Introduction}

Microscopic fungi from the genus Fusarium are an integral element of soil microflora but are also encountered in underground and aerial parts of plants, plant debris and other organic substrates $[1,2]$. They induce numerous economically important diseases of crops, e.g. head blight in cereals, Fusarium root rot, corn cob fusariosis, etc., substantially reducing the cereal crop yields and the quality of produce [3-6]. The combined effect of several Fusarium species, infecting more than one crop at the same time and producing one or more mycotoxins, is an interesting example of biodiversity. During the vegetation and storage, cereals could be attacked by multiple fungal species forming the epiphytic microflora. Fusarium species, causing infections of cereal crops (corn, wheat, barley, oat, etc.) have a dominant position [5,7]. 
A plant may become infected with Fusarium spp. at any stage of its development, depending on both the plant species and the infecting species. Therefore, having in mind that most Fusarium species possess specific mycotoxigenic profiles, the early and accurate identification of Fusarium spp. is essential for predicting the potential risk of infection and prevention of mycotoxin occurrence along the food chain. The unambiguous identification of mycotoxigenic Fusarium species is still an exceptionally critical problem because the number of species in the genus is constantly changing according to various taxonomic systems. A considerable number of Fusarium spp. classifications demonstrated a lack of uniform criteria for identifying species affiliation. Thus, various names are used for the same species or different species are grouped under the same name. The issue is further complicated by the fact that Fusarium species are exceptionally variable and often mutate. Also, many toxigenic strains degenerate and lose toxin-producing ability when stored on artificial nutrient media in laboratory conditions. The information from species recognition criteria used so far morphological, biological, and phylogenetic species recognition specific for a given region rarely matches [8-10]. The main approach to classification of Fusarium spp., which is still independently used, is the morphological one although, in many instances, information provided only by it could be misleading. The reason is the occurrence of some processes in fungi as hybridization, cryptic speciation and convergent evolution [11-13]. The independent application of the morphological recognition of microfungi is often limited with regard to a number of morphological features. For example, cultures of some endosymbiotic fungi that routinely produce secondary metabolites, do not exhibit spores. In other spore-forming species, a considerable variation in the shape and size of some asexual structures (conidia, spores) exists within the same strain [14]. In these cases, the exact identification of species affiliation of the respective strain is very challenging. To avoid such limitations, it is advised to combine the morphological approach for identification of microscopic fungi with other contemporary methods of analysis allowed to obtain accurate and consistent results. To this end, a number of mycologists recommend using methods based on DNA sequencing for identification of fungi with high genetic diversity level [15-17].

The application of molecular techniques for species recognition of mould fungi dates back to more than two decades with nuclear ribosomal operon primers described by White et al. (1990) [18] including three nuclear ribosomal genes encoding a large ribosomal unit (nrLSU-26S or 28S), a small subunit (nrSSU-18S) and an internal transcribed region functioning as a spacer (ITS1, 5.8, ITS2). The identification of molds on the basis of sequencing, also known as DNA barcoding, is based on the utilization of the nrDNA-ITS region. The meeting of the international consortium of mycologists held in 2011 in Amsterdam has proposed 6 DNA regions from the fungal genome and one of them, namely the ITS region, was officially approved for DNA barcoding marker [19]. Additional fungal studies have provided support for the ITS region as a suitable fungal barcode [20-22]. Moreover, most metagenomic studies of fungi are using the ITS region in their studies to identify fungi using modern sequencing technology, which can aid in rapid identification of fungi $[23,24,25,26,27,28]$. This method for molecular genetic identification requires comparing the studied sequence to the respective reference sequence from a database, e.g. GenBank at the National Center for Biotechnology Information, the European Nucleotide Sequence Archive of the European Molecular Biology Laboratory etc. [29-30].

The ribosomal spacer has a number of advantages which make it a convenient target region for molecular genetic identification of fungi, namely: 1) easy amplification with universal primers, 
complementary to gene sequences encoding ribosomal DNA molecules; 2) the ITS region is represented as multiple copies (over 200) in the genome as a mosaic of permanent (conserved) and diverse nucleotide sequences allowing for easy amplification even from small amounts diluted DNA or degraded samples; 3 ) numerous studies demonstrated that the ITS region had a high degree of fungal interspecies variability [31-33]. A report in the Journal of Natural Products, containing summarised data for the use of different methods for fungal analysis and the distribution of published research in 2010-2016 showed an increasing tendency for utilisation of nrDNA-ITS as a marker of early fungal species recognition $(42 \%)$, as compared to independently applied morphological (8\%) and other methods [10].

The aim of the present study was to perform molecular identification of Fusarium spp. strains, isolated from wheat originated from Stara Zagora region, Bulgaria with regard to their taxonomy and phylogenesis by sequencing of the nrDNA-ITS region.

\section{Materials and Methods}

\section{Experimental sample collection}

A total of 60 wheat samples were analyzed in order to establish the degree of contamination and species from genus Fusarium. The wheat samples were collected from different locations in Stara Zagora region (lands of villages: Zagore, Badeshte, Pastren, Mogila, Sarnevo, Hrishteni, Dalboki, Oriacohvitca, Sabrano, Khan Asparuch, Malka Vereia, Rakitnica, Yavorovo, Vinarovo, Svoboda, Zetyovo, Spasovo, Sredetc, Znamenosetc, Merichlery, Galabovo) according to ISO 24333:2010 [34] at the period July-August 2018 from storage facilities. From these samples, 12 microscopic fungal strains from the genus Fusarium were isolated. The isolates were identified to the genus level in the microbiology lab, according to the taxonomy of Gerlach and Nirenberg (1982) [35].

\section{Nutrient media and reagents}

Samples for mycological examination were inoculated on Czapek agar (MERCK®) containing: saccharose - 30 g; NaNO3 - 3.0 g; K2HPO4 - 1.0 g; MgSO4.7H2O - 0.5 g; KCl - 0.5 g; FeSO4.7H2O $0.01 \mathrm{~g}$; agar - $15 \mathrm{~g}$; distilled water $-1000 \mathrm{ml}$ ( $\mathrm{pH}=7.2$ ). A selective nutrient medium (SNA) recommended by Nirenberg (1976) [36], containing: K2HPO4 - 1.0 g; KNO3 - 1.0 g; MgSO4.7H2O $0.500 \mathrm{~g}$; glucose $-0.2 \mathrm{~g}$; saccharose $-0.2 \mathrm{~g}$; agar $-20 \mathrm{~g}$; distilled water $1000 \mathrm{ml}$. (pH=5.6), was used for pure culture obtaining. All used reagents were analytical and reagent grade.

\section{Mycological examination}

One hundred whole grains were collected from each sample after being superficially sterilized with $70 \%$ ethanol for $5 \mathrm{~min}$, followed by three items of washing with distilled water to eliminate surface microflora. Grains were placed in 4 Petri dishes $(\mathrm{d}=15 \mathrm{~cm})$ (25 grains in each) on Czapek Dox medium (MERCK $®$ ) (Dimitrov 1983). Inoculated plates were incubated at $22-25^{\circ} \mathrm{C}$ for 7 days, (BSS 11374/86). After the incubation, the number of grown colonies belonging to the genus Fusarium was counted.

The affiliation of colonies to the genus level was done according to Dimitrov (1983) [37] using the identification keys. The genus level identification included culture examination (colony diameters, 
colony characters), following by preparation of wet mounts for microscopy by cutting out with inoculating needle a small portion of the colony, which includes sporing structures. Isolation of pure cultures was performed by use of single spore techniques described by Pitt \& Hocking (2009) [38]. A suspension of spores was prepared in a $10 \mathrm{ml}$ sterile water blank so that it contains 1-10 spores per low-power (10X) microscope. The water agar medium plates were poured with $0.1 \mathrm{ml}$ of suspension and incubated for $18-20 \mathrm{~h}$ at $25^{\circ} \mathrm{C}$. Obtained single spore cultures were transferred onto a selective medium (SNA) (Nirenberg 1976 [36]) and incubated at $25^{\circ} \mathrm{C}$ for 7 days.

\section{DNA extraction and PCR amplification}

Genomic DNA from Fusarium spp. strains was isolated from ingrown on SNA plates mycelium with diameter $1 \pm 2 \mathrm{~cm}$, initially ground with quartz sand to degrade the polysaccharide cellular wall. For isolation of DNA from samples, the Plant DNA Preparation Kit (Jena Bioscience, Germany) was used according to manufacturer's instructions. The concentration of isolated genomic DNA in each sample was measured on a NanoView Plus (GE Healthcare) spectrophotometer at a wavelength of 260-280 $\mathrm{nm}$. The DNA concentration in all samples was adjusted to10 $\mathrm{ng} / \mu \mathrm{l}$, in a working volume of $60 \mu \mathrm{l}$ and verified by electrophoresis on $1 \%$ agarose gel (TopVision agarose, Fermentas, USA). Samples with isolated genomic DNA were stored at $-18^{\circ} \mathrm{C}$.

For PCR amplification of the nrDNA-ITS region, the primer pair (Bioneer, Korea), proposed by White et al. (1990) [18] was used. PCR reactions were run with genomic DNA amount of $200 \mathrm{ng}$. To this end, 1.5mM Red Taq Master mix reaction mixture (VWR, Denmark) with a total volume of $50 \mu 1$ was prepared. Amplification was performed on a thermocycler VWR Doppio (General Electric, UK). The optimum annealing temperature of primers $\left(52.5^{\circ} \mathrm{C}\right)$ was preliminarily determined by repeated PCR amplification of the same sample within the temperature range $49.3^{\circ} \div 60.7^{\circ} \mathrm{C}$.

The obtained PCR products were identified by horizontal electrophoresis on $2 \%$ agarose gel diluted in $1 \times$ TBE buffer, at $80 \mathrm{~V} / 60 \mathrm{~min}$. Agarose gels with incorporated products were observed on MiniBis photo documentation system under UV light by means of transilluminator (ECX-15M Bio-Imaging Systems), after staining with $10000 \times$ GelRed $^{\mathrm{TM}}$ Nucleic Acid Stain (Biotium Inc, USA). Obtained products were visualized through GelCapture image acquisition software. The measurement of fragments' length was verified with DNA marker (GeneRuler ${ }^{\mathrm{TM}}$, 50 bp., Fermentas).

\section{Direct sequencing of the region $r D N A-I T S$}

A total of 12 samples (PCR products) were sequenced for the studied nrDNA-ITS region in the Bioneer research lab, South Korea. Sequences for each of samples, provided by Bioneer, were used for subsequent software processing. Bioneer Sequencing Service is a fast and highly reliable service performed on the ABI 3730XL DNA Analyzer, which provides high-quality of sequence analysis data (Phred Score $(\mathrm{QV}): \geq 20$, Guaranteed read lengths: $\geq 700 \mathrm{bp}$ ) within $24 \mathrm{hr}$ from the arrival of sample. We also used Bioneer's oligo synthesis supports for ITS custom primer.

\section{Statistical Analysis}

Identification of isolates was performed by comparison of each sequence to respective reference one from the NCBI database through Nucleotide Blast (https://blast.ncbi.nlm.nih.gov/Blast.cgi). DNA Sequence Assembler v4. (2013) was used for conversion of sequences from „txt" to „Fasta“ format. The established sequences of analysed samples were edited, assembled and aligned by the CLUSTALW tool, using the MEGA7 v.0.26. software [39]. The same software was used to determine 
genetic distances between studied fungal isolates and phylogenetic tree was reconstructed by the Neighbour-joining method [40-41].

\section{Results}

\section{- Mycological examination analysis}

Mycological examinations of 60 wheat samples harvested in 2018 yielded 12 strains belonging to species from the genus Fusarium. The main mycoflora of wheat samples determined by morphological examination of the fungal isolates (colony characters and microscopically observation of fruit bodies, phiallide, absence of chlamydspores etc.) comprised 9 genera microscopic fungi: Fusarium spp., Aspergillus spp., Penicillium spp., Alternaria spp., Mucor spp., Rhizopus spp., Cladosporium spp., Rhizoctonia spp. and Nigrospora spp. The contamination rate of individual samples with Fusarium spp. ranged from $0-4 \%$, and rates for other genera (Alternaria and Mucor) varied within broader ranges. For the rest of genera, contamination rates varied insignificantly. It should be noted that during 2017-2018, a period characterized by low soil and air humidity during the vegetation of wheat, contamination with different members of the genus Fusarium was expectedly low. Out of the 30 studied wheat samples, $12(40 \%)$ were positive for Fusarium spp., whereas the other $18(60 \%)$ not contaminated with fungi from this genus.

\section{- Data analysis from PCR amplification of fungal isolates}

The rDNA-ITS region of 12 fungal strains isolated from wheat was successfully amplified by gradient PCR and fragments with the expected length were obtained (Figure 1). The amplicon lengths for Fusarium isolates varied from 515 to $557 \mathrm{bp}$. The highest amplified product length was that of Alternaria infectoria - $578 \mathrm{bp}$, while that of Chaetomium spp. was $551 \mathrm{bp}$.

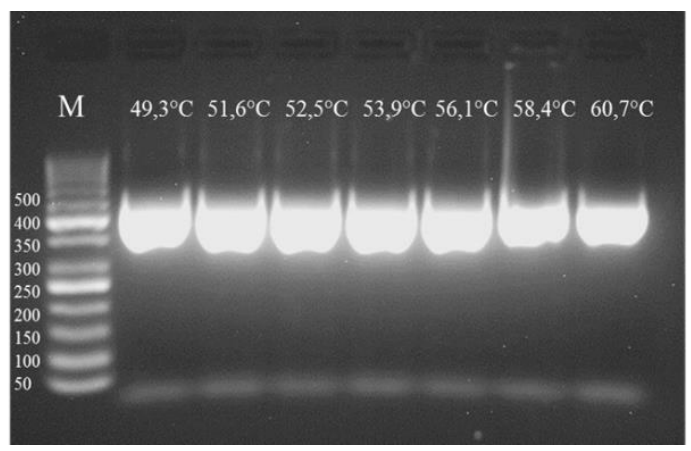

Figure 1. Electrophoregram of PCR fragments on $2 \%$ agarose gel

M - Marker (DNA Ladder, $50 \mathrm{bp}$ ), Lanes 2-8 - PCR samples of nrDNA-ITS region in studied fungal isolates, obtained at different annealing temperatures

\section{- Data analysis from nrDNA-ITS region sequencing in Fusarium spp. samples}

The analysis comprised 12 nucleotide sequences. The sequence alignment was done according to the CLUSTALW algorithm allowing for the elimination of all missing nucleotides (gaps). The fragment with a total of 12 aligned sequences (Figure 2) shows clearly the nucleotide identity of 10 of studied fungal isolates (samples 1, 2, 4, 5, 6, 7, 9, 10, 11 and 12) and nucleotide variation for samples 3 and 8 . 


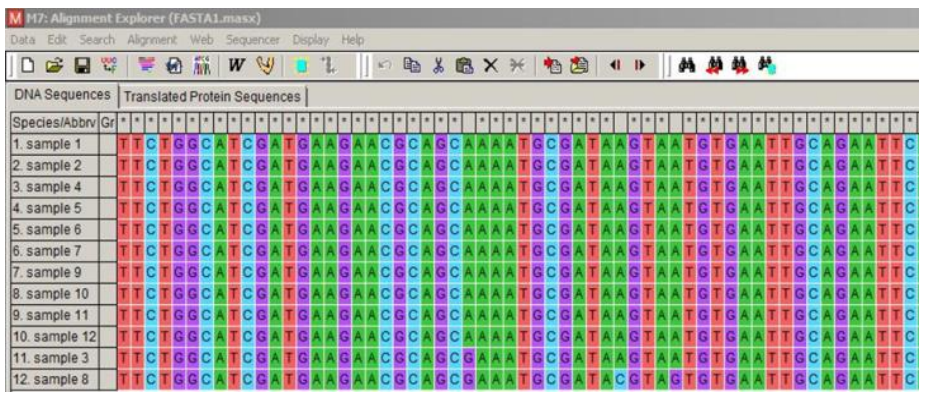

Figure 2. Fragment with rDNA-ITS region sequences from 12 Fusarium spp. isolates in MEGA 7, aligned by CLUSTALW; * A, G, C, T- nucleotides

The obtained nucleotide sequences were submitted in the GenBank under the accession numbers: MN982764 for Fusarium spp., MN982876 for Chaetomium spp. and MN982879 for Alternaria infectoria.

- Data analysis from the identification of fungal isolates

The identification of isolates of studied molds was performed by Nucleotide Blast in NCBI, by comparison of studied sequences to reference sequences from the database. The species affiliation of fungal isolates is presented in Table 1.

Table 1. Affiliation of the studied fungal isolates on the basis of nrDNA-ITS region sequencing

\begin{tabular}{rllll}
\hline $\begin{array}{l}\text { Sample } \\
\text { № }\end{array}$ & Species affiliation & Strain / isolate & Fragment size, bp & $\begin{array}{l}\text { ID number in } \\
\text { GenBank, NCBI }\end{array}$ \\
\hline 1 & Fusarium tricinctum & WS11790 & 535 & JX406512.1 \\
2 & Fusarium poae & 5331 & 515 & MG736211.1 \\
3 & Chaetomium sp. & C7-3 & 551 & MG818934.1 \\
4 & Fusarium poae & 5331 & 515 & MG736211.1 \\
5 & Fusarium tricinctum & GMG105 & 534 & KJ598871.1 \\
6 & Fusarium tricinctum & WS11791 & 538 & JX406511.1 \\
7 & Fusarium proliferatum & JUF0013 & 557 & MH368119.1 \\
8 & Alternaria infectoria & A-1-6 & 578 & KT223326.1 \\
9 & Fusarium graminearum & FSHG & 552 & KU939070.1 \\
10 & Fusarium tricinctum & WS11791 & 538 & JX406511.1 \\
11 & Fusarium tricinctum & WBS019 & 533 & KU350729.1 \\
12 & Fusarium equiseti & s079 & 523 & HQ649909.1 \\
\hline
\end{tabular}

Among Fusarium isolates, the F. tricinctum was the prevailing species $(\mathrm{n}=5)$, followed by $F$. poae $(\mathrm{n}=$ 2). F. proliferatum, F. graminearum and F. equiseti were presented with a single isolate, which agrees with previous information provided by Beev et al. (2011) [42] about the presence of F. equiseti in Bulgarian wheat. The isolate No 3 was recognized as Chaetomium sp, and isolate No 8 - as Alternaria infectoria. The identity achieved by the analysis is $99 \%$, indicating high reliability of the applied method. 


\section{- Analysis of genetic distances and phylogenesis of fungal isolates}

Genetic distances among studied fungal isolates were calculated by MEGA7 v. 0.26 software, on the basis of the number of nucleotide substitutions in analyzed sequences. The lowest genetic distance (0.004) was demonstrated between F. tricinctum isolates, followed by that between F. poae (0.007). The F. proliferatum strain which is differentiated as an independent branch of the phylogenetic tree, was more genetically similar to $F$. tricinctum than to F. poae, while F. equiseti and F. graminearum exhibited higher genetic identity with $F$. poae. The cited values are depicted on Figure 3.

On the basis of genetic distance values, the phylogenetic tree (Figure 3) was reconstructed using the Neighbour-joining method.

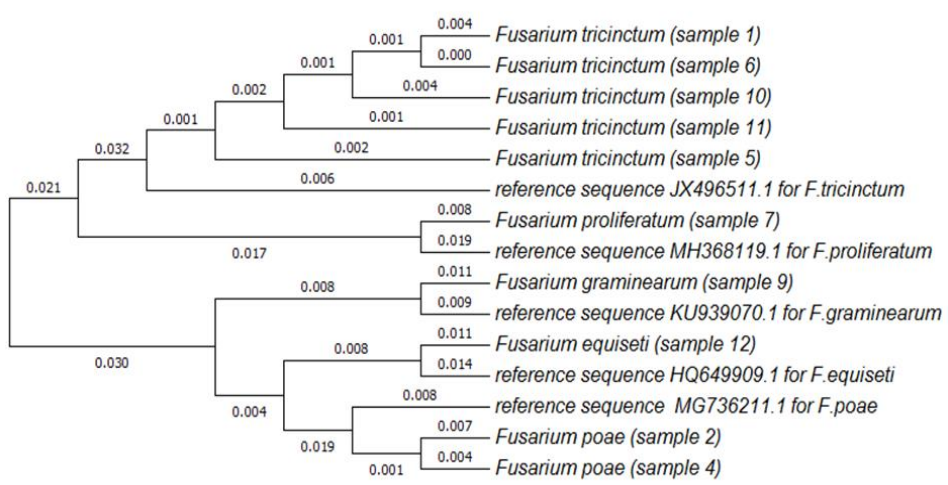

Figure 3. Neighbour-joining - dendrogram built on the base of obtained sequences of the region rDNA-ITS of 10 Fusarium isolates from wheat samples in accordance with their reference sequences obtained from NCBI

The length of phylogenetic tree branches is given with a figure corresponding to genetic distances between studied strains. Neighbour-joining - dendrogram was built on the base of obtained sequences of the region rDNA-ITS of 10 Fusarium isolates from wheat samples in accordance with their reference sequences obtained from NCBI database. The dendrogram (Figure 3) showed that fungal isolates were grouped in two main clusters - one comprising 5 F. tricinctum isolates and $F$. proliferatum, and another including 3 isolates of F. equiseti, F. graminearum and F. poae strains.

\section{Discussion}

Different Fusarium species causing wilt diseases in different plants have been also characterized by comparing the ITS region of nonpathogenic and pathogenic species via RFLP assay. For example, The ITS (internal transcribed spacer) region of 12 Fusarium isolates belonging to the section Elegans, Liseola, Mortiella, Discolor, Gibbosum, Lateritium and Sporotrichiella have been amplified by Data et al. (2011) [43] based on universal ITS primers (ITS-1 and ITS-4). The amplified products (ranged from 522 to $565 \mathrm{bp}$ ) have been digested with seven restriction enzymes. A dendrogram derived from PCR-RFLP analysis of the rDNA region has divided the Fusarium isolates into three major groups. In conclusion, the authors pointed out that the assessment of molecular variability based on rDNA RFLP clearly indicated that Fusarium species are heterogeneous and most of the forma speciales have close evolutionary relationships. In order to use the ITS molecular data to identify phytopathogenic form fungus Fusarium, several restriction enzymes specific for each special form have been selected by Suda et al. (2000) [44] from the mapping data. Schoch et al. (2012) pointed out 
that among the regions of the ribosomal cistron, the internal transcribed spacer region has the highest probability of successful identification for the broadest range of fungi, with the most clearly defined barcode gap between inter- and intra-specific variation.

\section{Conclusions}

The obtained results in the present study could be affirmed that the primary microflora of the examined samples comprised a total of 9 genera microscopic fungi: Fusarium spp., Aspergillus spp., Penicillium spp., Alternaria spp., Mucor spp., Rhizopus spp., Cladosporium spp., Rhizoctonia spp. and Nigrospora spp. Among Fusarium isolates, the F. tricinctum was prevailing $(\mathrm{n}=5)$, followed by F. poae $(\mathrm{n}=2)$. F. proliferatum, F. graminearum and F. equiseti were presented with a single isolate. The trend for the occurrence of F. equiseti on wheat was confirmed. The lowest genetic distance (0.004) was detected between F. tricinctum isolates. The F. proliferatum strain was separated as an independent branch of the phylogenetic tree, showing higher genetic similarity to F. tricinctum than to F. poae. The sequencing analysis allowed determinating phylogenetic relationships between studied isolates, which formed 2 clearly distinct phylogenetic tree branches. The results indicated clearly that using genomic rDNA-ITS region was suitable for early detection of wheat seedlings' contamination for the prevention of Fusarium infections.

Author Contributions: Georgi Beev collected the fungal isolates and performed mycological examination. Deyana Gencheva performed DNA analysis. The authors wrote the paper together.

Funding: This work was supported by the Bulgarian Ministry of Education and Science under the National Research Programme "Healthy Foods for a Strong Bio-Economy and Quality of Life" approved by DCM \# 577/17.08.2018.

Conflicts of Interest: All data generated or analysed during this study are included in this published article.The authors declare that they have no competing interests.

\section{References}

Author 1, A.; Author 2, B. Title of the chapter. In Book Title, 2nd ed.; Editor 1, A., Editor 2, B., Eds.; Publisher: Publisher Location, Country, 2007; Volume 3, pp. 154-196.

Author 1, A.; Author 2, B. Book Title, 3rd ed.; Publisher: Publisher Location, Country, 2008; pp. 154-196.

1. Marasas W.F., Nelson P.E., Toussoun T.A. Toxigenic Fusarium Species: Identity and Mycotoxicology, The Pennsylvania State University Press: University Park, PA, 1984.

2. Burgess L.W., Summerell B.A. Mycogeography of Fusarium: survey of Fusarium species in subtropical and semi-arid grassland soils from Queensland, Australia. Mycologycal Research, 1992, 96, 780-784.

3. Parry D.W., Jenkinson P., Mcleod L.. Fusarium ear blight (scab) in small grain cereals - a review. Plant pathology, 1995, 44, 207-238.

4. Draganova Ts., Daskalov P., Tsenkova R., Atanassova S., Veleva-Doneva P. Recognition of Fusarium diseased corn kernels using NIR spectroscopy and Neuro-Fuzzy classifier. Proceedings of Third IFAC International Conference, Agricontrol, Kyoto, Japan, 2010, 3, 219-224.

5. Beev G., Denev S., Bakalova D.. Zearalenone-producing activity of Fusarium graminearum and Fusarium oxysporum isolated from Bulgarian wheat, Bulgarian Journal of Agricultural Science, 2013, 19, 255-259. 
6. Daskalov P, Draganova Ts, Veleva-Doneva P, Beev G. Quality assessment of corn kernels (healthy and infected by fusarium) via a decision tree. Journal of Science and Technology, Natural \& Mathematical science, 2013, 3, 237-242. (Bg)

7. Glenn A.E. Mycotoxigenic Fusarium species in animal feed. Animal Feed Science and Technology, 2007, 137, 213-240.

8. Desjardins A. E. Fusarium mycotoxins: chemistry, genetics and biology. St Paul, Minnesota: Phytopathology, 2006, 260.

9. Edel V., Gautheron N., Recorbet G., Alabouvette C. Genetic diversity of Fusarium oxysporum populations isolated from different soils in France. FEMS Microbiology Ecology, 2001, 36, 61-71.

10. Raja H.A., Miller A.N., Pearce C.J., Oberlies N.H. Fungal identification using molecular tools: a primer for the natural products research community. Journal of Natural Products, 2017, 80, 756-770.

11. Brun S., Silar P. In Evolutionary Biology - Concepts, Molecular and Morphological Evolution, Editor Pontarotti P., Springer: Berlin Heidelberg, 2010, pp. 317-328.

12. Hughes K.W., Petersen, R.H., Lodge D.J. Bergemann S.E., Baumgartner K., Tulloss R.E., Lickey, E., Cifuentes J. Evolutionary consequences of putative intra-and interspecific hybridization in agaric fungi. Mycologia, 2013, 105, 1577-1594.

13. Lücking, Dal-Forno M., Sikaroodi M., Gillevet P.M., Bungartz F., Moncada B., Yánez-Ayabaca A., Chaves J.L., Coca, L.F., Lawrey J.D. A single macrolichen constitutes hundreds of unrecognized species. Proceedings of the National Academy of Sciences of the United States of America, 2014, 111, 11091-11096.

14. Ko T.W., Stephenson S.L., Bahkali A.H., Hyde K.D. From morphology to molecular biology: can we use sequence data to identify fungal endophytes? Fungal Diversity, 2011, 50, 113-120.

15. Bridge P., Spooner B., Roberts P. The impact of molecular data in fungal systematics. Advances in Botanical Research, 2005, 42, 33-67.

16. Hibbett D.S., Ohman A., Glotzer D., Nuhn M., Kirk P., Nilsson R.H. Progress in molecular and morphological taxon discovery in Fungi and options for formal classification of environmental sequences. Fungal Biology Reviews, 2011, 25, 38-47.

17. Taylor J.W., Hibbett D.S. Toward sequence-based classification of fungal species. IMA Fungus, 2013, 4, 33-34.

18. White T.J., Bruns T., Lee S., Taylor J. Amplification and direct sequencing of fungal ribosomal RNA genes for phylogenetics. In PCR Protocols, Editors Innis M.A., Gelfand D.H., Sninsky J.J., White T.J., Academic Press, San Diego, 1990, pp. 315-322.

19. Schoch C.L., Seifert K.A., Huhndorf S., Robert V., Spouge J.L., Levesque C.A. \& Fungal Barcoding Consortium. Nuclear ribosomal internal transcribed spacer (ITS) region as a universal DNA barcode marker for Fungi. Proceedings of the National Academy of Sciences, 2012, 109, pp. 6241-6246.

20. Seena S., Pascoal, C., Marvanová L. \& Cássio F. DNA barcoding of fungi: a case study using ITS sequences for identifying aquatic hyphomycete species. Fungal Diversity, 2010, 44, 77-87.

21. Dentinger B.T., Didukh, M.Y. \& Moncalvo J.M. Comparing COI and ITS as DNA barcode markers for mushrooms and allies (Agaricomycotina). PLoS one, 2011, 6, 9.

22. Kelly L.J., Hollingsworth, P.M., Coppins, B.J., Ellis, C.J., Harrold, P., Tosh, J. \& Yahr R. DNA barcoding of lichenized fungi demonstrates high identification success in a floristic context. New Phytologist, 2011, 191, 288-300.

23. O'Brien H.E., Parrent J.L., Jackson J.A., Moncalvo J.M. \& Vilgalys R. Fungal community analysis by large-scale sequencing of environmental samples. Applied and Environmental Microbiology, 2005, 71, 5544 $55-50$.

24. Bellemain E., Carlsen T., Brochmann C., Coissac E., Taberlet P., Kauserud H. BMC Microbiology, 2010, 10 (1), 189.

25. Nilsson R.H., Tedersoo L., Lindahl B.D., Kjøller R., Carlsen T., Quince C., Abarenkov K., Pennanen T., Stenlid J., Bruns T., Larsson K.H., Koljalg U., Kauserud H. Towards standardization of the description and publication of next-generation sequencing datasets of fungal communities. New Phytologist, 2011, 119, 314318.

26. Roslin A., Cox F., Cruz-Martinez K., Ihrmark K., Grelet G.A., Lindahl B.D., Menkis A., James T.Y. Science, 2011, 333, 876-879.

27. Wang Z., Nilsson R.H., Lopez-Giraldez F., Zhuang W.Y., Dai Y.C., Johnston P.R., Townsend J.P. PLoS One, 2011, 6, 19039 . 
28. Ihrmark K., Bödeker I.T., Cruz-Martinez K., Friberg H., Kubartova A., Schenck J., Strid Y., Stenlid J., Brandström-Durling M., Clemmensen K.E., Lindahl B.D. FEMS Microbiology Ecology, 2012, 82, 666-677.

29. Robert V., Cardinali G., Stielow B., Vu T., dos Santos F.B., Meyer W., Schoch C. Fungal DNA barcoding. Molecular Biology of Food and Water Borne Mycotoxigenic and Mycotic Fungi, 2015, 37-56.

30. Yahr R., Schoch C.L., Dentinger B.T. Scaling up discovery of hidden diversity in fungi: impacts of barcoding approaches. Philosophical Transactions of the Royal Society B: Biologycal Sciences, 2016, 371, 20150336.

31. Gardes M., Bruns T.D. ITS primers with enhanced specificity for Basidiomycetes - application to the identification of mycorrhizae and rusts. Molecular Ecology, 1993, 2, 113-118.

32. Nilsson R.H., Ryberg M., Abarenkov K., Sjökvist E., Kristiansson E. The ITS region as a target for characterization of fungal communities using emerging sequencing technologies. FEMS Microbiology Letters, 2009, 296, 97-101.

33. Blaalid R., Kumar S., Nilsson R.H., Abarenkov K., Kirk P.M., Kauserud H. ITS1 versus ITS2 as DNA metabarcodes for fungi. Molecular Ecology Resources, 2013, 13, 218-224.

34. Cereals and cereal products - Sampling (ISO 24333:2009).

35. Gerlach, W. and Nirenberg, H. The genus Fusarium - a pictorial atlas. - Mitt. Biol. Bundesanst. Land Forstwirtsch., Berlin-Dahlem,1982, 209, 1-406.

36. Nirenberg H.I. Untersuchungen Liber die morphologische und biologische Differenzierung in der Fusarium-Sektion Liseola. Mitt. Biol. Bundesanst. Landu. Forstwirtsch. Berlin-Dahlem, 1976, 169, 11-17.

37. Dimitrov M., Nicolcheva M., Engibarova P., Drenska E., Popova T. Fusarium fungal species isolated from wheat, maize and beans. Plant science, 1983, 20, 120-125 (Bg).

38. Pitt, J.I. and Hocking A.D. Fungi and Food Spoilage, 3rd ed., Springer Dordrecht Heidelberg London New York, 2009, DOI 10.1007/978-0-387-92207-2.

39. Kumar S., Stecher G., Tamura K. MEGA7: molecular evolutionary genetics analysis version 7.0 for bigger datasets. Molecular Boilogy and Evolution, 2016, 33, 1870-1874.

40. Saitou N., Nei M. The neighbor-joining method: a new method for reconstructing phylogenetic trees. Moecular Biology and Evolution, 1987, 4, 406-425.

41. Tamura K., Nei M., Kumar S. Prospects for inferring very large phylogenies by using the neighbor-joining method. Proceedings of the National Academy of Sciences, 2004, 101, 11030-11035.

42. Beev G., Denev S., Pavlov D. Occurrence and distribution of Fusarium species in wheat grain. Agricultural Science and Technology, 2011, 3, 165-168.

43. Datta S., Choudhary R.G., Shamim Md, Dhar V. Polymorphism in the internal transcribed spacer (ITS) region of the ribosomal DNA among different Fusarium species. Archives of Phytopathology and Plant Protection, 2011, 44 (6), 558-566.

44. Suga H., Hasegawa T., Mitsui H., Kageyama K., Hyakumachi M. Phylogenetic analysis of the phytopathogenic fungus Fusarium solani based on the rDNA-ITS region. Mycologycal Research, 2000, 104, 1175-1183.

45. Schoch C.L., Seifert K.A., Huhndorf S., Robert V., Spouge J.L., Levesque C.A., Fungal Barcoding Consortium. Nuclear ribosomal internal transcribed spacer (ITS) region as a universal DNA barcode marker for Fungi. Proceedings of the National Academy of Sciences, 2012, 109, 6241-6246. 